\title{
Validación española del Cuestionario de Motivos para el Juego (GMQ) en población general
}

\author{
Carola Cerdà Salom \\ al189477@uji.es \\ SARA NEBOt IBÁÑEZ \\ snebot@uji.es \\ Daniel Campos Bacas \\ camposd@uji.es \\ Soledad Quero Castellano \\ squero@uji.es
}

\section{Resumen}

Introducción: El juego patológico en población joven es un problema que está cobrando una importancia cada vez mayor. Además, el porcentaje de adolescentes con este problema en los últimos años es más elevado que el de los adultos. Es importante realizar una adecuada evaluación de este problema en esta población. El objetivo de este estudio es llevar a cabo la validación española del Cuestionario de Motivos para el Juego (GMQ; Gambling Motives Questionnaire; Stewart y Zack, 2008) en población general joven. Método: La muestra incluía 106 participantes (33 mujeres y 73 hombres) con edades de entre 17 y 23 años. Todos los participantes cumplimentaron el GMQ junto con otros cuestionarios relacionados. El GMQ consta de 15 ítems que evalúan las razones por las cuales las personas deciden jugar y tiene 3 subescalas: ENH (mejora emociones positivas), SOC (motivos sociales) y COP (reducir emociones negativas). Resultados: Se obtuvieron buenos coeficientes de consistencia interna para las 3 subescalas y una excelente fiabilidad test-retest. El análisis factorial exploratorio arrojó una estructura de 3 factores que estaban intercorrelacionados y, también, correlacionaron positivamente con la Escala Relacionada con el Juego. La subescala COP del GMQ correlacionó negativamente con la subescala general de la Escala de Regulación de Afecto Negativo. Conclusiones: La validación del GMQ en población joven supone una aportación relevante en Psicología Clínica. Es necesario contar con instrumentos basados en la evidencia que permitan diferenciar los jugadores problemáticos de los que no lo son y que sean sensibles al cambio tras programas de prevención o tratamiento.

Palabras clave: evaluación, validación, motivos de juego, juego problemático, juego patológico. 


\begin{abstract}
Introduction: Pathological gambling in young people is a problem that is increasingly important. In addition, the percentage of adolescents with this problem in the last years is higher than that of adults. It is important to conduct a suitable assessment of this problem in this population. The aim of this study is to carry out the Spanish validation of the Gambling Motives Questionnaire (GMQ, Stewart y Zack, 2008), in general young people. Method: The sample consisted of 106 young participants from the general population (33 women and 73 men), aged between 17 and 23 years. All participants completed the GMQ along with other self-report related measures. The GMQ consists of 15 items that assess the reasons why people decide to play and has 3 subscales: ENH (enhance positive emotions), SOC (social motives) and COP (decrease negative emotions). Results: Good internal consistency coefficients for the 3 subscales and excellent test-retest reliability were obtained. Exploratory factor analysis showed a structure of 3 factors that were inter-correlated and, also all of them correlated positively with The Gambling Related Scale. Conclusions: The subscale COP of the GMQ correlated negatively with the general subscale of the Scale of Negative Affect Regulation. The validation of the GMQ in young people represents a significant contribution in Clinical Psychology. It is necessary to have evidence-based measures to differentiate problem gamblers from those who are not problematic and to be sensitive to change after a prevention or treatment program.
\end{abstract}

Keywords: evaluation, validation, gambling motives, problematic gambling, pathological gambling.

\title{
Introducción
}

Los estudios epidemiológicos realizados en diferentes países muestran una incidencia cada vez mayor del juego patológico en la población general que coincide con el incremento en la legalización de las apuestas y el aumento del tipo de juegos en los últimos años. Por lo que se refiere al juego patológico en la población joven, es un problema que está cobrando una importancia cada vez mayor. En Estados Unidos, se encontró una prevalencia de juego patológico en estudiantes de enseñanza secundaria y universitaria de entre $4 \%$ y $8 \%$ (Lesieur y Rosenthal, 1991). En España, en un estudio sobre el juego problemático en las máquinas tragaperras, realizado por Fisher (1993) con jóvenes de 11 a 16 años de A Coruña y Gijón, se encontró una prevalencia de $2 \%$ y 1,6 \%, respectivamente. Por último, Becoña (1997) en una muestra de adolescentes encontró una prevalencia de $2,4 \%$ de probables jugadores patológicos.

Además de constituir un problema prevalente en este sector de la población, el porcentaje de adolescentes con ludopatía en estos últimos años es más elevado que el de los adultos (Muñoz-Molina, 2008). Por este motivo, es de gran importancia tanto la realización de programas de prevención dirigidos a esta población como poder contar con instrumentos de medida basados en la evidencia para realizar una adecuada evaluación de este problema.

Uno de los cuestionarios con el que contamos, y que es objeto de este estudio, es el Cuestionario de motivos para el juego (GMQ: Gambling Motives Questionnaire; Stewart y Zack, 2008). Este cuestionario evalúa las razones por las cuales las personas deciden jugar. Es una adaptación de la Medida tridimensional de motivos para beber (Three-dimensional measure of 
drinking motives; Cooper, Russell y Skinner, 1992). Stewart y Zack (2008) administraron el GMQ a una muestra de 193 personas seleccionadas a partir de sus puntuaciones en el Cuestionario de juego patológico de South Oaks (sogs: South Oaks Gambling Screen; Lesieur y Blume, 1987). Se obtuvieron dos grupos, uno de 154 probables jugadores patológicos y otro de 39 jugadores no patológicos, todos ellos menores de 19 años. El análisis factorial arrojó 3 factores: ENH (incrementar emociones positivas), SOC (refuerzo positivo externo) y COP (reducir o evitar emociones negativas). Los resultados mostraron una buena consistencia interna para los 3 factores del cuestionario y se observó intercorrelación entre los factores. También se observaron diferencias de sexo en el grupo de jugadores patológicos probables; los hombres obtuvieron puntuaciones más altas en el factor $\mathrm{ENH}$, mientras que en las mujeres la puntuación más elevada se encontró en el factor COP. En el grupo de jugadores no patológicos no se observaron diferencias de sexo.

Debido a la utilidad y las buenas propiedades psicométricas que presenta este instrumento y, teniendo en cuenta que en la actualidad no se dispone de ninguna validación de este cuestionario en población española, el principal objetivo de este trabajo es llevar a cabo la validación española del Cuestionario de motivos para el juego (Stewart y Zack, 2008) en población joven.

\section{Método}

\section{Participantes}

La muestra estaba compuesta por 106 participantes (33 mujeres y 73 hombres) de población general joven. Todos ellos eran residentes en la Comunidad Valenciana: 56 provenían de un centro de deportistas de alto rendimiento, 25 eran alumnos de $2^{\circ}$ de Bachillerato de un Instituto de Educación Secundaria y los 25 restantes eran estudiantes de la Universitat Jaume I y de la Universitat de València. La media de edad fue de 19.066 (d. t. =1,96), oscilando el rango entre 17 y 23 años.

\section{Medidas}

El Cuestionario de Motivos para el Juego (GMQ) fue administrado a los participantes junto con otros instrumentos de autoinforme que se describen a continuación:

- Cuestionario de Motivos para el Juego (GMQ; Gambling Motives Questionnaire; Stewart y Zack, 2008). Consta de 15 ítmes que evalúan los motivos que tienen los jugadores a la hora de jugar a los juegos de azar en una escala de tipo Likert de 4 puntos que va de 1 («nunca o casi nunca») hasta 4 («casi siempre»). Está compuesto por 3 factores: «mejora de emociones positivas» (ENH: ítems 3, 6, 9, 12, 15 ), «refuerzo positivo externo» (Soc: $1,4,7,10,13$ ) y «reducir o evitar de emociones negativas» (COP: $2,5,8,11,14)$. Tiene buenas propiedades psicométricas en población joven de habla inglesa (Stewart y Zack, 2008).

- Escala Relacionada con el Juego (GRCS; The Gambling related Scale; Raylu y Oei, 2004). Evalúa los pensamientos que tienen los participantes a la hora de jugar a los diferentes juegos de azar en una escala tipo Likert que va de 1 («muy en desacuerdo») a 7 («muy de acuerdo»). Está compuesta por 23 ítems divididos en 5 subescalas: expectativas de juego, ilusión de control, control predictivo, incapacidad para detener 
el juego y sesgo interpretativo. Ha mostrado tener buenas propiedades psicométricas (Raylu y Oei, 2004).

- Escala de Regulación del Afecto Negativo (ERAN) (Catanzaro y Mearns, 1990). Evalúa la capacidad de regulación emocional que tienen las personas cuando se sienten mal de ánimo. Consta de 30 afirmaciones y se compone de una puntuación total y 3 subescalas: la subescala general (evalúa la capacidad de aliviar el afecto negativo mediante diferentes estrategias); la subescala cognitiva (evalúa pensamientos y estrategias cognitivas) y la subescala conductual (evalúa acciones que influyen en el estado de ánimo negativo).

- Inventario de Depresión de Beck (BDI-II: Beck Depression Inventory; Beck, Steer y Brown, 1996; Adaptación española por Sanz, Navarro y Vázquez, 2003). Se trata de un instrumento de autoinforme compuesto de 21 ítems diseñado para evaluar la sintomatología depresiva en adultos y adolescentes con una edad a partir de 13 años. Este instrumento cuenta con buenas propiedades psicométricas en población española (Sanz, Navarro y Vázquez, 2003).

\section{Procedimiento}

Tras llevar a cabo el proceso de retrotraducción de la versión original del GMQ, se hizo llegar el protocolo de evaluación para el juego a toda la muestra de estudio. En algunos casos se cumplimentaron vía online (mediante la aplicación Survey Monkey) y en otros en papel.

\section{Resultados}

En primer lugar, se analizó la consistencia interna de las tres subescalas del GMQ mediante el coeficiente de Cronbach, obteniendo un coeficiente de 0,87 para la subescala ENH, de 0,79 para la subescala soc y de 0,87 para la subescala cop. En la tabla 1 se presentan las correlaciones de cada ítem con el total. Por otra parte, la fiabilidad test-retest para una parte de la muestra $(\mathrm{N}=25)$ utilizando el coeficiente de dos mitades de Guttman fue de 0,995 .

En cuanto al análisis factorial, siguiendo a los autores originales (Stewart y Zack, 2008) $y$, debido a que las subescalas del GMQ estaban intercorrelacionadas, se realizó el análisis factorial mediante el método de extracción de componentes principales con rotación Oblimin. Se obtuvo una estructura de 3 factores (ver tabla 1) que explicaban el 68,54\% de la varianza total. El factor 1 «Afrontamiento y mejora de emociones», explicaba el 50,65\% de la varianza y se componía de 9 ítems $(2,3,5,6,9,11,12,14,15)$. El factor 2, «Motivos sociales», lo formaban 4 ítems $(1,4,10,13)$, que explicaban el $9,98 \%$ de la varianza. Por último, el factor 3 , «Motivos para aumentar la autoconfianza y seguridad en uno mismo ante relaciones sociales», lo formaban los ítems 7 y 8 , que explicaban el 7,89 \% de la varianza. El coeficiente de Cronbach para estos tres factores fue de $0,91,0,79$ y 0,67 , respectivamente. 
Tabla 1

Estadísticos descriptivos, alfa de Cronbach, correlación ítem-total y cargas factoriales para el GMQ

\begin{tabular}{|c|c|c|c|c|c|c|}
\hline Ítem & $\begin{array}{l}\text { Media } \\
\text { (d. t.) }\end{array}$ & $\begin{array}{c}\text { Alfa de Cronbach } \\
\text { si se elimina } \\
\text { el ítem }\end{array}$ & $\begin{array}{l}\text { Correlación } \\
\text { ítem-total }\end{array}$ & Factor 1 & Factor 2 & Factor 3 \\
\hline $\begin{array}{l}\text { 1. Como una forma } \\
\text { de celebración }\end{array}$ & $1,45(0,82)$ & 0,709 & 0,677 & & 0,576 & \\
\hline 2. Para relajarte & $1,28(0,63)$ & 0,843 & 0,684 & 0,846 & & \\
\hline $\begin{array}{l}\text { 3. Porque te gusta } \\
\text { la sensación }\end{array}$ & $1,54(0,87)$ & 0,832 & 0,706 & 0,537 & 0,444 & \\
\hline $\begin{array}{l}\text { 4. Porque es lo que } \\
\text { hacen la mayoría } \\
\text { de tus amigos } \\
\text { cuando estáis } \\
\text { juntos }\end{array}$ & $1,61(0,88)$ & 0,743 & 0,585 & & 0,921 & \\
\hline $\begin{array}{l}\text { 5. Para olvidar tus } \\
\text { preocupaciones }\end{array}$ & $1,29(0,65)$ & 0,843 & 0,688 & 0,722 & & \\
\hline 6. Porque es excitante & $1,37(0,65)$ & 0,825 & 0,766 & 0,882 & & \\
\hline 7. Para ser sociable & $1,15(0,41)$ & 0,797 & 0,418 & & & 0,873 \\
\hline $\begin{array}{l}\text { 8. Porque te sientes } \\
\text { más confiado } \\
\text { o seguro de ti } \\
\text { mismo }\end{array}$ & $1,14(0,48)$ & 0,880 & 0,515 & & & 0,675 \\
\hline $\begin{array}{l}\text { 9. Para lograr sensa- } \\
\text { ciones fuertes }\end{array}$ & $1,39(0,81)$ & 0,833 & 0,698 & 0,784 & & \\
\hline $\begin{array}{l}\text { 10. Porque es algo } \\
\text { que hago en oca- } \\
\text { siones especiales }\end{array}$ & $1,66(0,88)$ & 0,733 & 0,612 & & 0,687 & \\
\hline $\begin{array}{l}\text { 11. Porque te ayuda } \\
\text { cuando te sientes } \\
\text { nervioso o depri- } \\
\text { mido }\end{array}$ & $1,25(0,60)$ & 0,811 & 0,815 & 0,724 & & \\
\hline $\begin{array}{l}\text { 12. Porque es diverti- } \\
\text { do }\end{array}$ & $1,80(1,03)$ & 0,853 & 0,667 & 0,505 & 0,479 & \\
\hline $\begin{array}{l}\text { 13. Porque hace } \\
\text { que una reunión } \\
\text { social sea más } \\
\text { divertida }\end{array}$ & $1,46(0,83)$ & 0,739 & 0,592 & 0,454 & 0,435 & \\
\hline $\begin{array}{l}\text { 14. Para animarte } \\
\text { cuando estás } \\
\text { de mal humor }\end{array}$ & $1,34(0,67)$ & 0,819 & 0,777 & 0,569 & & 0,428 \\
\hline $\begin{array}{l}\text { 15. Porque te hace } \\
\text { sentir bien }\end{array}$ & $1,30(0,62)$ & 0,841 & 0,693 & 0,778 & & \\
\hline
\end{tabular}


En cuanto a las diferencias de sexo, la prueba $t$ de Student no arrojó diferencias estadísticamente significativas entre hombres y mujeres en ninguna de las 3 subescalas del GMQ.

Por último, se llevaron a cabo correlaciones de Pearson bivariadas de las 3 escalas del GMQ con otras medidas que pudieran estar más o menos relacionadas con la conducta de juego: la GRCS (tanto con su puntuación total como con sus 5 subescalas), el BDI y la ERAN (tanto con su puntuación total como con sus 3 subescalas). También se llevaron a cabo correlaciones entre las escalas del GMQ. Como se puede observar en la tabla 2, los resultados mostraron que las 3 subescalas del GMQ correlacionaron de forma positiva y significativa $(p<0,01)$ con el GRCS, tanto con su escala global como con todas sus subescalas. En cuanto al BDI, no se observó ninguna correlación significativa con ninguna de las subescalas del GMQ. Por lo que se refiere a la ERAN, se observó una correlación negativa y significativa $(p<0,01)$ entre su subescala general (mide la capacidad de aliviar el afecto negativo mediante diferentes estrategias) y la subescala COP del GMQ. Asimismo, se obtuvieron correlaciones positivas y significativas $(p<0,01)$ entre las 3 subescalas del GMQ.

Tabla 2

Correlaciones entre el GMQ y otras medidas

\begin{tabular}{lccc}
\hline & GMQ-ENH & GMQ-SOC & GMQ-COP \\
\hline GMQ-ENH & 1 & & \\
GMQ-SOC & $0,701^{* *}$ & 1 & \\
GMQ-COP & $0,752^{* *}$ & $0,629^{* *}$ & 1 \\
GRCS-PC & $0,493^{* *}$ & $0,421^{* *}$ & $0,444^{*}$ \\
GRCS-GE & $0,712^{* *}$ & $0,542^{* *}$ & $0,734^{* *}$ \\
GRCS-IC & $0,382^{* *}$ & $0,487^{* *}$ & $0,496^{* *}$ \\
GRCS-IS & $0,525^{* *}$ & $0,427^{* *}$ & $0,625^{* *}$ \\
GRCS-IB & $0,633^{* *}$ & $0,526^{* *}$ & $0,543^{* *}$ \\
GRCS-total & $0,630^{* *}$ & $0,549^{* *}$ & $0,635^{* *}$ \\
BDI-II & 0,023 & 0,018 & 0,060 \\
ERAN (general) & $-0,168$ & $-0,185$ & $-0,209^{* *}$ \\
ERAN (cognitiva) & $-0,126$ & $-0,123$ & $-0,143$ \\
ERAN (conductual) & 0,088 & 0,040 & $-0,065$ \\
ERAN (total) & $-0,097$ & $-0,096$ & $-0,157$ \\
\hline
\end{tabular}

Nota: ** $p<0,01 ;{ }^{*} p<0,05$

Nota: GMQ-ENH: GMQ, subescala «incrementar emociones positivas; GMQ-SOC: GMQ, subescala «refuerzo positivo externo»; GMQCOP: GMQ, subescala «reducir emociones negativas»; GRCS-PC: escala relacionada con el juego, subescala control predictivo; GRCS-GE: subescala expectativas de juego; GRCS-IC: subescala ilusión de control; GRCS-IS: subescala incapacidad para detener el juego; GRCS-IB: subescala sesgo interpretativo; GRCS-total: puntuación total; BDI-II: inventario de depresión de Beck; ERAN: Escala de regulación del afecto negativo. 


\section{Discusión y conclusiones}

El objetivo de este trabajo fue realizar la validación española del Cuestionario de motivos para el juego (GMQ) en población joven. Los resultados obtenidos confirmaron que las tres subescalas del GMQ presentaban una buena consistencia interna. Por otra parte, se obtuvo una excelente estabilidad temporal.

Con respecto al análisis factorial, al igual que en la validación realizada por los autores originales (Stewart y Zack, 2008), se obtuvieron 3 factores. Sin embargo, aunque el porcentaje de varianza total explicada fue bastante similar a la original $(68,54 \%$ frente a $67,2 \%$ ), la estructura hallada en este trabajo mostró diferencias a la obtenida en la validación original. El factor 1 (afrontamiento y mejora de emociones), explicaba un porcentaje mayor de la varianza $(50,65 \%$ frente a $49,3 \%)$ y la mayoría de ítems incluídos entre los factores 1 (ENH) y 3 (COP) de la validación original saturaron claramente en este primer factor (ítems 2, 3, 5, 6, 9, 11, 12, 14 y 15), a excepción del ítem 8. Respecto al factor 2 (motivos sociales) obtenido en el presente estudio, explicó un procentaje algo mayor de varianza que el de la validación original $(9,98 \%$ frente a 9,1\%). Este factor incluía los ítems 1, 4, 10 y 13, que coinciden con el factor 2 de la validación original, a excepción del ítem 7. Por último, el factor 3 (aumento de autoconfianza y seguridad en uno mismo ante relaciones sociales), quedó compuesto por 2 ítems (7 y 8) y explicó el 7,89 \% de la varianza, mientras que en la validación original explicaba el $8,8 \%$. Estas diferencias podrían deberse al hecho de que el presente estudio se realizó con una muestra de población general joven sin problemas de juego, mientras que la muestra utilizada por los autores originales estaba formada por población también joven pero muchos de ellos eran jugadores patológicos probables.

Por lo que se refiere a los resultados sobre las diferencias en función del sexo, se obtuvieron resultados similiares a los hallados por Stewart y Zack (2008), ya que estos autores tampoco encontraron diferencias entre hombres y mujeres en el grupo de jugadores no patológicos.

Por último, los resultados de validez convergente mostraron que, por lo que se refiere al GRCS, se confirmó que este cuestionario y los factores que lo componen correlacionaban de forma positiva y significativa con las 3 subescalas del GMQ. El GRCs evalúa los pensamientos de los participantes a la hora de jugar a los diferentes juegos de azar. Así, es esperable que exista una relación significativa y positiva entre este cuestionario y el GMQ, ya que a mayor nivel de pensamientos irracionales relacionados con el juego, mayores motivos hacia el juego. Por otra parte, la subescala general de la ERAN, que evalúa la capacidad de aliviar el afecto negativo mediante diferentes estrategias, correlacionó de forma negativa y significativa con la subescala COP del GMQ. Una mayor puntuación en este instrumento indica una mayor regulación emocional. De este modo, los sujetos que puntúan alto en esta subescala del GMQ utilizan el juego como una estrategia para aliviar sus emociones negativas, por lo que parecen tener escasas capacidades para regular su afecto negativo. Finalmente, aunque cabría esperar una correlación positiva entre el BDI-II con la subescala COP del GMQ, no es de extrañar no haberla encontrado, ya que el presente estudio se realizó con una muestra de población general joven sin síntomas de depresión ni problemática asociada al juego.

En conclusión, los resultados obtenidos demuestran que la versión española del GMQ cuenta con buenas propiedades psicométricas para evaluar los motivos de juego en población joven española. Es necesario contar con instrumentos de evaluación basados en la evidencia que permitan diferenciar los jugadores problemáticos de los que no lo son y que sean sensibles al cambio tras un programa de prevención o tratamiento. Por último, es importante señalar que el presente trabajo consiste en una validación psicométrica que está en progreso, por 
lo que en próximos estudios será necesaria una ampliación de la muestra, aumentando su tamaño y utilizando también población clínica para obtener conclusiones más firmes.

\section{Referencias bibliográficas}

Beck, A. T., Steer, R. A. y Brown, G. K. (1996). Manual for the Beck Depression Inventory II. Psychologica Corp, San Antonio, TX.

Becoña, E. (1997). Pathological gambling in Spanish children and adolescents: An emerging problem. Psychological Reports, 81, 275-287.

Catanzaro, S. J. y Mearns, J. (1990). Measuring Generalized Expectancies for Negative Mood Regulation: Initial Scale Development and Implications. Journal of personality assessment, 54, 546-563.

Cooper, M. L., Russell, M. y Skinner, J. B. (1992). Development and validation of three-dimensional measure of drinking motives. Psychological Assessment, 4, 123-32.

Fisher, S. (1993). El impacto del juego de máquinas tragaperras legales para niños sobre el juego y el juego patológico en adolescentes. El caso del Reino Unido. Psicología Conductual, 1, 351-359.

Leisieur, H. R. y Blume, S. B. (1987). The South Oaks Gambling Screen (sogs): a new instrument for the identification of pathological gamblers. American Journal of Psychiatry, 144, 1184-8.

Leisieur, H. R. y Rosenthal, R. J. (1991). Pathological gambling: A review of the literature. Journal of gambling Studies, 7, 5-39.

Muñoz-Molina, Y. (2008). Meta-análisis sobre el juego patológico. Revista de Salud Pública, 10, 150-159.

Raylu, N. y Oei, T. P. (2004). The Gambling Related Cognitions Scale (GRCS): development confirmatory factor validation and psychometric properties. Addiction, 99, 775-769.

Sanz, J., Navarro, M. E. y Vázquez, C. (2003). Adaptación española del Inventario para la Depresión de Beck-II (BDI-II): Propiedades psicométricas en estudiantes universitarios. Análisis y modificación de conducta, 29, 239-288.

Stewart, S. H. y Zack, M. (2008). Development and psychometric evaluation of a three-dimensional Gambling Motives Questionnaire. Addiction, 103, 1110-1117. 